\title{
SOME RECENTLY DEVELOPED FRACTIONATION PROGEDURES AND THEIR APPLIGATION TO PEPTIDE AND PROTEIN HORMONES
}

\author{
J. Porath \\ Biokemiska Institutionen, Universitet Uppsala, Sverige
}

\section{INTRODUGTION}

In $1921 \mathrm{H}$. M. Evans injected a homogenate of anterior pituitary lobe tissues into rats and found that they gained in weight. From the time of this discovery twenty-three years passed before Evans, in collaboration with $\mathrm{Li}$, reported the isolation of a growth hormone. Even today $\mathrm{Li}$ and his collaborators are making considerable efforts to improve the purification methods for this growth hormone. I think this fact illustrates very well the difficulties encountered in attempts to fractionate protein and peptide hormones. However, the time and labour spent on such projects often prove to be profitable. Some of the achievements in the field represent landmarks in the history of biochemistry. Two outstanding pioneering contributions which might be mentioned are the elucidation of the amino-acid sequence of insulin and the synthesis of vasopressin and oxytocin. Evidently in these cases, as in so many others, the isolation had to precede chemical analysis and synthesis. Without pure hormones only incomplete information can be obtained about the chemical structure; without extensive knowledge of the chemistry of the hormones their mode of action cannot be explained in chemical or physical terms. After these introductory remarks I hope to have made it clear that elaboration of powerful fractionation procedures is an important occupation.

Within the last ten years my colleagues and I have studied the melanophore-stimulating hormone, vasopressin, oxytocin, corticotropin and its releasing factor, and recently the thyrotropic hormone. The biological assays have always been made by our colleagues in other laboratories while fractionation, chemical and physical characterization have been carried out in our institute at Uppsala. Two fractionation methods have been extensively used: zone electrophoresis and gel filtration. In addition to these procedures, counter-current extraction, ion-exchange, liquidliquid partition and charcoal chromatography have also been employed. In the fractionation schemes the various methods have been used in sequences that have proved to be extremely effective. Of all these methods gel filtration is the most informative since it often makes possible an estimation of the molecular size. Gel filtration is a very versatile method that already has been applied to problems in many fields both in organic chemistry and biochemistry. I believe it is appropriate to discuss this method in more detail now since we have studied it extensively recently. 


\section{J. PORATH}

\section{THEORETICAL ASPECTS}

If pieces of a gel are placed in a solution of glucose and sucrose these solutes will diffuse into the gel at a rate which depends on the molecular dimensions not only of the sugars but also of the gel matrix. Glucose and sucrose differ in their diffusion coefficients in free solution and their differential diffusion rate will be further enhanced by the selective influence of the steric hindrance within the gel. Such differences in diffusion rates may be utilized for fractionation as proposed by Biget ${ }^{1}$. Selectivity may also be based on the extent of penetration under equilibrium or pseudo-equilibrium conditions; this is the case in gel filtration ${ }^{2}$.

A xerogel, i.e. a gel imbibing large quantities of solvent, contains regions differing in accessibility for solutes. The accessibility depends on the sterical relationships between the solute molecules and the gel network. Small molecules may completely penetrate the gel particles while large molecules and particles are effectively excluded. Molecules of intermediate size have access to comparatively open regions of the gel. The molecular size range for penetrability of the solutes can be varied by changing the structure of the gel. A compact gel will thus exclude substances which may easily penetrate a more expanded kind of gel.

We shall now attempt to derive an expression for the distribution of solutes between the gel and the outer solution. Let us assume that the interior of the gel can be reached only by those solute molecules, moving towards the free liquid-gel boundary, which do not collide with the atoms lining the openings of the network. The openings at any place on the gel surface are likely to have very irregular perimeters. Likewise, the linings of the alveolar spaces or cavities must be irregular in shape. Since there is no preference as to shape of the cavities we may treat them as being sterically identical from a statistical viewpoint. We assume the cavities to be conical in shape and of a certain average size with a diameter of $A$ and a total depth of $H$. If a molecule, having an effective radius of $R$, can enter the openings and reach a maximal depth of $h$ it is easily seen that the centre of the molecule is bound to distribute freely within the volume $v$ defined by

$$
v=\frac{h \pi}{12}(A-2 R)^{2}
$$

The whole cavity space is

$$
\boldsymbol{V}=\frac{H \pi}{12} A^{2}
$$

The distribution coefficient is proportional to the ratio between $v$ and $V$ if no adsorption takes place. Hence

$$
K_{\mathrm{d}}=k \frac{v}{V}=k \frac{h}{H}\left(\frac{A-2 R}{A}\right)^{2}
$$

But

$$
\bar{H}=\frac{A-2 R}{A}
$$


SOME REGENTLY DEVELOPED FRACTIONATION PROCEDURES

and consequently

$$
K_{\mathrm{d}}=k\left(1-\frac{2 R}{A}\right)^{3}
$$

( $k$ is a proportionality coefficient).

$A^{3}$ and the volume of solvent in the gel, "solvent regain" $S_{\mathrm{r}}$, must presumably be closely interrelated. Let us assume that

$$
A^{3} \sim S_{\mathrm{r}}-\alpha
$$

where $\alpha$ is a correction term introduced to account for a part of the solvent which is closely associated with the gel matrix and not exchangeable for solutes.

For flexible polymers consisting of identical segments the gyration radius is proportional to the square root of the molecular weight ${ }^{3}, \mathrm{M}$ :

$$
R \sim \mathrm{M}^{\frac{1}{2}}
$$

Equations (4), (5) and (6) yield

$$
K_{\mathrm{d}}=k\left[1-k_{1} \frac{\mathrm{M}^{\frac{1}{2}}}{\left(S_{\mathrm{r}}-\alpha\right)^{1 / 3}}\right]^{3}
$$

( $k$ is a proportionality coefficient).

The parameters $A, H$ and $h$ used in the derivation of equation (7) are of a rather fictitious character. The proposed concept must, therefore, be tested with experimental data. $K_{\mathbf{d}}$ must be fairly accurately determined and this can be done by the chromatographic technique called gel filtration. The technical procedure is as follows:

$\mathrm{Gel}$ in the form of beads is suspended in a suitable solution and a column is prepared by letting the particles settle in a chromatographic tube. A sample solution is transferred to the top of the column and filtered into the bed. The solvent used in preparing the column is also used as eluent. The effluent solution is collected and analysed. Thus, gel filtration is a kind of elution chromatography in which the composition of the developer is kept constant. Molecular sieving takes place predominantly in the outer layer of the gel. Sufficiently small molecules only are able to penetrate into interior regions of the grains. When the solutes pass through the bed they are subjected to a kind of multiple dialysis which causes them to migrate with different speed. Thus, nonpenetrable solutes move outside the grains $\left(K_{d}=0\right)$ and appear after the passage of solvent volume, $V_{0}$, equal to the space of the voids. Other substances move more slowly and emerge from the column in the order of decreasing molecular size if adsorption and other discriminating separation factors can be neglected. The elution volume, $V_{e}$, depends on $K_{d}$ according to the following equation:

$$
V_{\mathrm{e}}=V_{\mathrm{o}}+K_{\mathrm{d}} V_{\mathrm{i}}
$$

where $V_{1}$ is the volume of the solvent in the gel. $V_{\mathrm{i}}$ may be expressed in terms of solvent regain, $S_{\mathrm{r}}$, and the amount of dry gel in the bed, $a$,

$$
V_{\mathbf{i}}=a S_{\mathrm{r}}
$$




\section{J. PORATH}

By combining equations (7), (8) and (9) we obtain

$$
\Delta V=V_{\mathrm{e}}-V_{0}=k a S_{\mathrm{r}}\left[1-k_{1} \frac{\mathrm{M}^{\frac{1}{2}}}{\left(S_{\mathrm{r}}-\alpha\right)^{1 / 3}}\right]^{3}
$$

The experimental data for low molecular weight dextran fraction published by Granath and Flodin ${ }^{4}$ have been used to test the validity of the equations. If the square root of the molecular weights are plotted against the cubic root of the distribution coefficients straight lines are obtained as expected from equation (7) (Figure 1). The lines have different slopes which

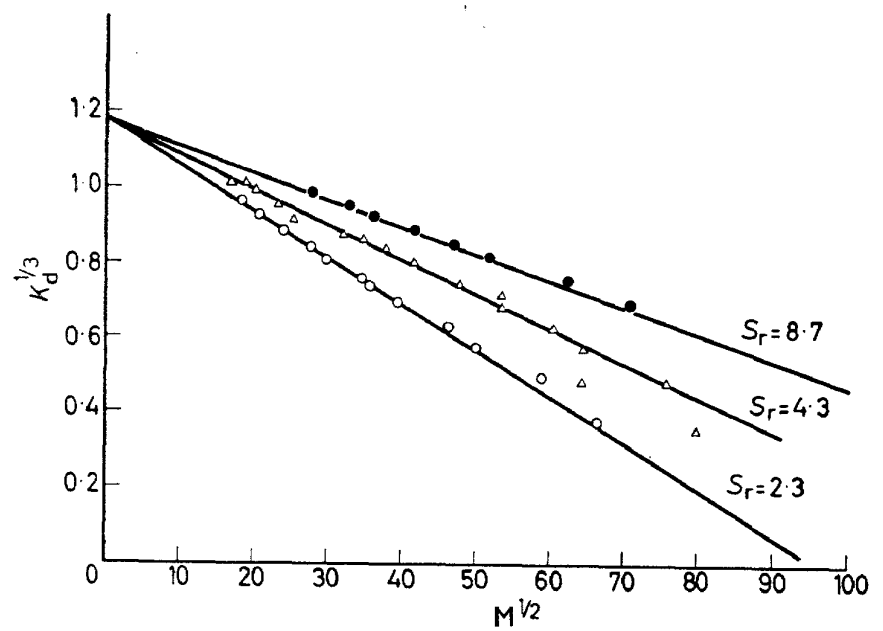

Figure 1. Illustration of the linear relationship between $K_{\mathrm{d}}{ }^{1 / 3}$ and $\mathrm{M}^{1 / 2}$ predicted by equation (7). The lines are drawn from the equation with $k=1 \cdot 64, k_{1}=0.012$ and $\alpha=0.8$. The dots and triangles are the experimental values reported by Granath and Flodin ${ }^{4}$

depend on the solvent regain values. The lines all converge to the same points on the ordinate axis as predicted. From the slopes and the ordinate at the intersection the coefficients $K_{1}$ and $k$ and the correction term $\alpha$ can be found. For the dextrans and the Sephadex gels $k$ is 1.64 and $k_{1}$ close to 0.012 . The value of $\alpha$ has been found to be 0.8 .

In Figure $2(a)$ is shown the fractionation of cellodextrins on Sephadex G 25 in distilled water. This gel filtration experiment was made by Flodin and Aspberg $^{5}$ when the technique was still in a rather undeveloped stage. Today, following these authors, it should be possible to increase the resolution and also to extend the effective fractionation to higher oligosaccharides. In Figure $2(b)$, equation (10) is used for cellodextrins to give the theoretical curve. The predicted and experimental retention values agree very well for cellotriose and the higher carbohydrates. The statistical treatment of segment distribution to give equation (6) is, of course, not valid for the lower oligomers. This may explain the poor agreement between the theoretical curve and the retention for glucose and cellobiose.

According to our experience the optimal distribution conditions for separation of two substances seem to be confined to $K_{d}$ values within the interval $0 \cdot 1-0 \cdot 9$. Equations (7) and (10) permit estimation of the proper 


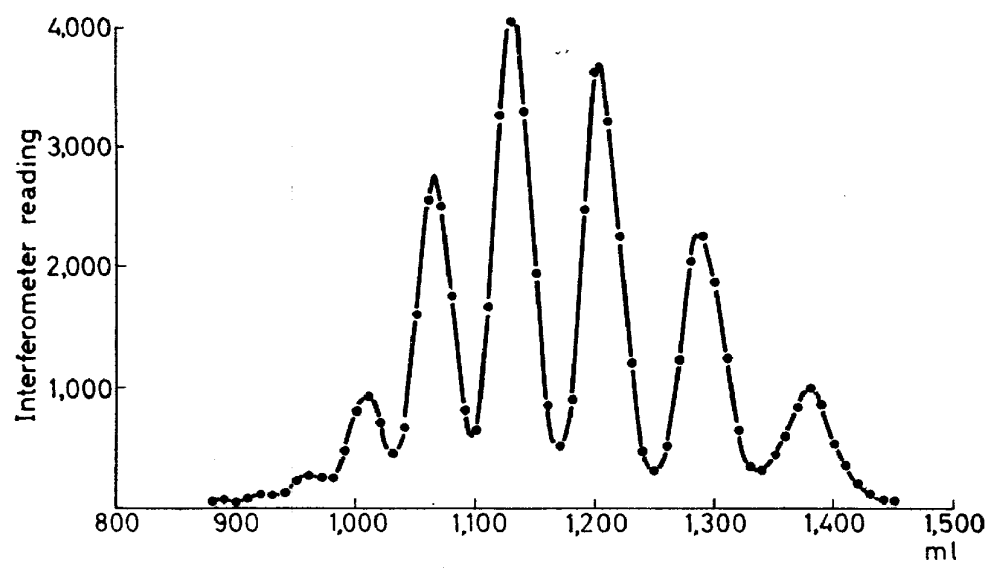

Figure 2(a). Gel filtration diagram of degradation products from cellulose. Gel: Sephadex G 25. From right to left: glucose, cellobiose, cellotriose $e t c$. according to Flodin and Aspberg ${ }^{5}$

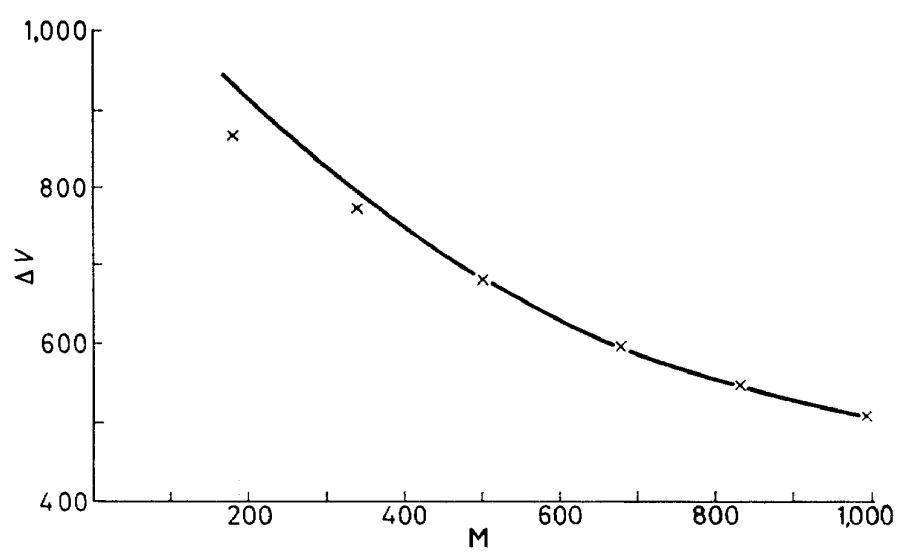

Figure $2(b)$. The retention values, $\Delta V$, as a function of the molecular weight. The curve has been calculated from equation $(10)$. The circles are the experimental values found from the filtration referred to in Figure 2(a)

solvent regain for a gel to be suitable for any molecular size range. The crosslinked dextrans now available can be used to fractionate mixtures of water-soluble substances of molecular weights from a few hundreds to well above 150,000 .

Equation (10) permits also the estimation of molecular weights by use of retention data from column experiments. The calculated molecular weight values should be interpreted cautiously since there are many pitfalls. For example, the coefficients $k$ and $K_{1}$ vary with the chemical nature of the gel and the extent of swelling. Gels of polyacrylamide and crosslinked dextran may have entirely different $k$ values for similar solvent regain. For the same kind of gel substance the extent of swelling may be widely different in a weak and a strong salt solution. Calibration should, therefore, be made 


\section{J. PORATH}

with due consideration for these circumstances. Completely wrong results may be obtained when the substances are adsorbed in addition to being sieved. Adsorption can be distinguished from molecular sieving by comparing the behaviour of the substances in gels of different solvent regain. Large deviations from equations (7) and (10) presumably reflect extensive adsorption, while small deviations indicate weak adsorption or perhaps unsymmetrical shape of the molecules, since in the latter case equations (1) and (6) may poorly approximate the true conditions.

We are now prepared to illustrate the method with some pituitary hormones.

\section{PURIFICATION OF VASOPRESSIN AND OXYTOCIN}

Vasopressin and oxytocin were among the first substances studied on dextran gel columns. They are strongly retarded on Sephadex G 25-the most extensively crosslinked dextran now on the market. Their $K_{\mathbf{d}}$ values are in fact close to unity ${ }^{6}$. This abnormal behaviour is caused by adsorption. Vasopressin and oxytocin are difficult to separate from aliphatic amino-acids and other unadsorbed low molecular weight compounds also present in the crude pituitary extract. A new approach was tried, by Chauvet, Lenci and Acher ${ }^{7}$ which took advantage of the fact that the hormones are bound to a carrier protein in the gland as a complex called neurophysin. We found later that neurophysin is excluded from. Sephadex G 25 and can, therefore, be freed from substances retarded on such a column (Figure $3(a)$ ). The carrier

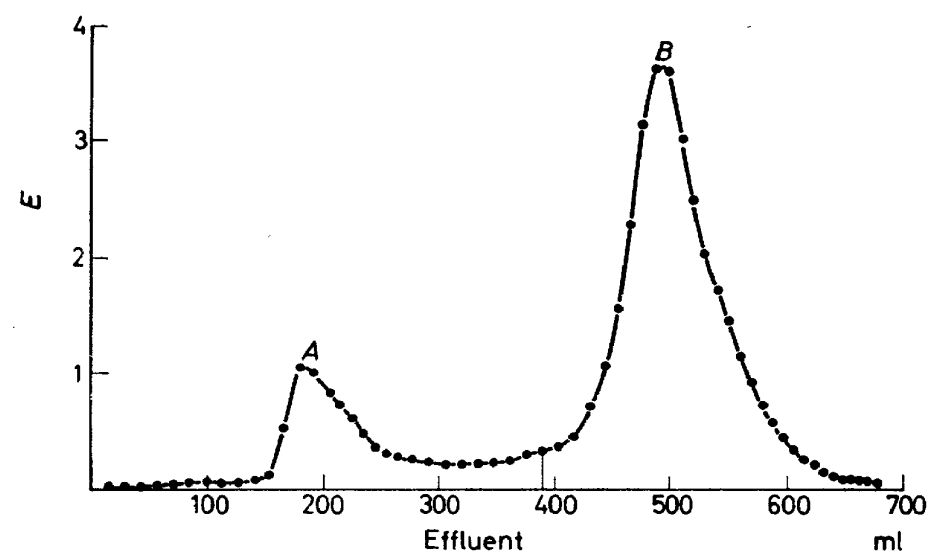

Figure 3(a). Gel filtration diagram of a crude extract from posterior lobes of hog pituitaries. The oxytocic and pressor activities are associated with the high molecular weight Fraction $A$

protein may then be split off by lowering the $\mathrm{pH}$ of the solution. When filtered through a G 25 column in acid medium, vasopressin and oxytocin will then separate from contaminating carrier and other proteins ${ }^{8}$ (Figure $3(b))$. 


\section{SOME RECENTLY DEVELOPED FRACTIONATION PROCEDURES}

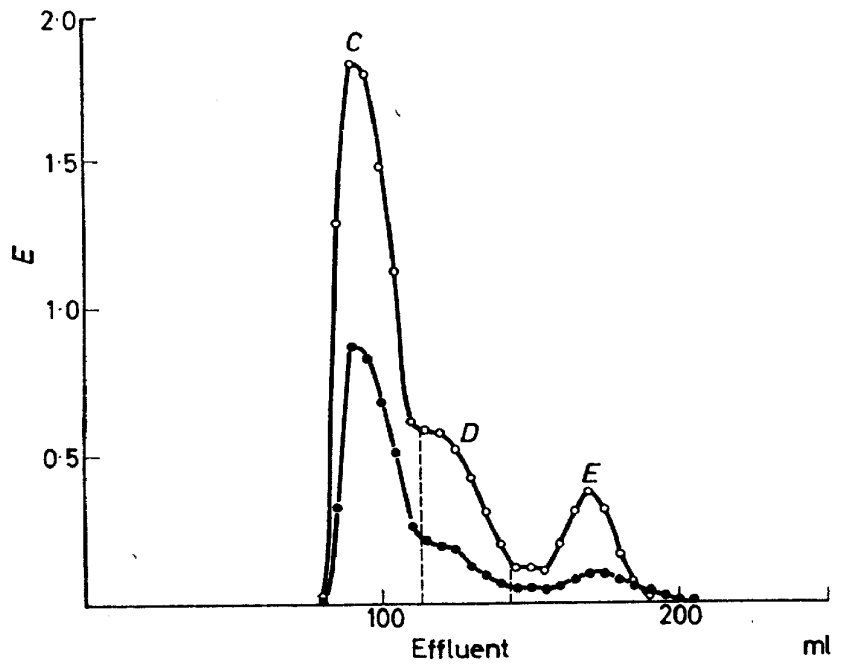

Figure $3(b)$. Refiltration of Fraction $A$ from Figure $3(a)$ in acid medium. The hormone activities are now recovered in the retained Fraction $E$. The filtrations illustrated in $(a)$ and

(b) have been made on the same kind of gel: Sephadex G 25

Increased efficiency may be obtained by performing the experiments in long columns. In Figure 4 is shown a gel filtration diagram of an oxytocin preparation. The main peak contained the oxytocin. The sharpness of the peaks illustrates the resolving power of a properly packed Sephadex column.

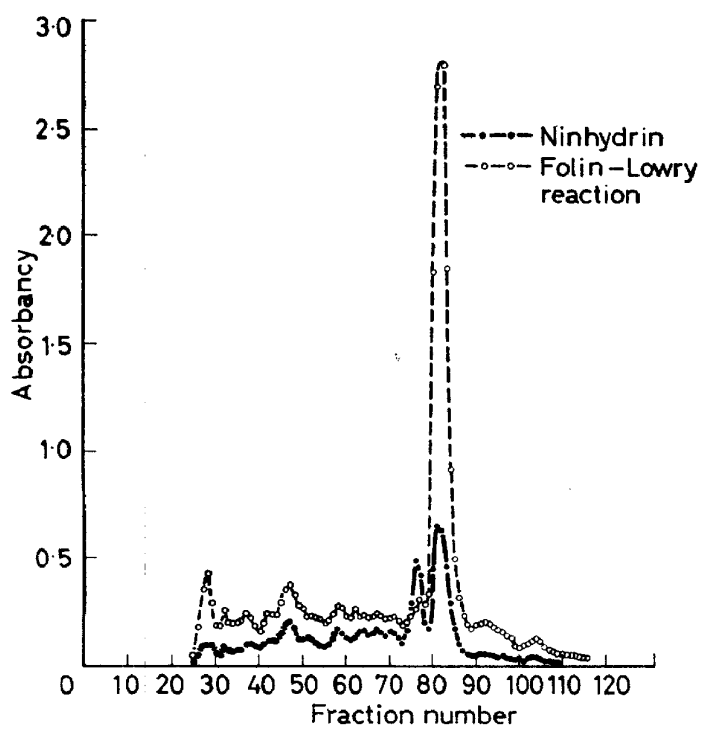

Figure 4. Fractionation of $200 \mathrm{mg}$ of partially purified oxytocin on Sephadex G $25^{11}$. Column dimensions : $2 \times 200 \mathrm{~cm}$; solvent: $0.2 \mathrm{M}$ acetic acid 


\section{J. PORATH}

While working on the fractionation of neurohypophyseal peptides we elaborated some other procedures which we believe ought to find widespread application. Since efficient exclusion could not be achieved in aqueous solution, mixed solvents were tried ${ }^{9}$. In the solvent systems chosen the extent of swelling of the gel substance was lower than in water. It was found that adsorption was suppressed. Vasopressin was found ${ }^{12}$ to be slightly retarded (peak 2, Figure 5) but moved well ahead of the amino-acids under

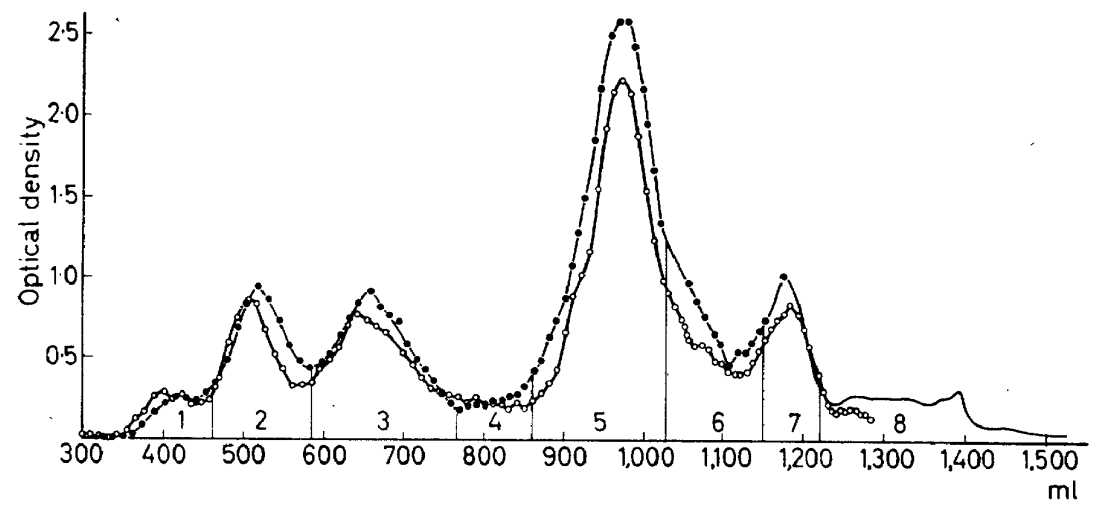

Figure 5. Gel filtration diagrams of two similar experiments of a preparation similar to Fraction $E$ in Figure $3(b)$. The experiments were made on Sephadex $\mathbf{G} 50$ in a solvent composed of 60 parts of acetic acid, 15 parts of pyridine and 25 parts of water

these conditions. The control of solvent regain by the choice of solvent composition may prove to be of great value for increasing the resolving power for mixtures of low molecular weight solutes. Organic solvents containing a high concentration of phenol have been used by Synge and Youngson to fractionate peptides from grass ${ }^{10}$. Such solvents permit effective splitting of complexes. The distribution in mixed solvents may sometimes also be governed by liquid-liquid partition.

If the methods just mentioned are supplemented by ion-exclusion on ionexchange columns ${ }^{9}$ pure hormones may easily be obtained ${ }^{11}$. Isolation of the hormones on a small scale can, therefore, be obtained within a day or two.

Ion exclusion on ionic derivatives of crosslinked dextran is a fractionation method which takes advantage of the combined effect of molecular sieving and electrostatic repulsion exerted by an ion exchanger on similarly charged solute ions. In pyridine-acetic acid, $\mathrm{pH} 5$, vasopressin and oxytocin are positively charged and so also are the ionizable groups of a diethylaminoethyl derivative of crosslinked dextran ("DEAE-Sephadex"). Figure 6 shows an ion-exclusion diagram obtained in an experiment with the retarded Fraction $E$ of Figure $3(b)$. Vasopressin is totally excluded while oxytocin is slightly retarded. Neutral contaminants move well behind the hormone peptides.

It is likely that the gel filtration methods could be used to advantage for working up reaction mixtures obtained in the final steps of the synthesis of 


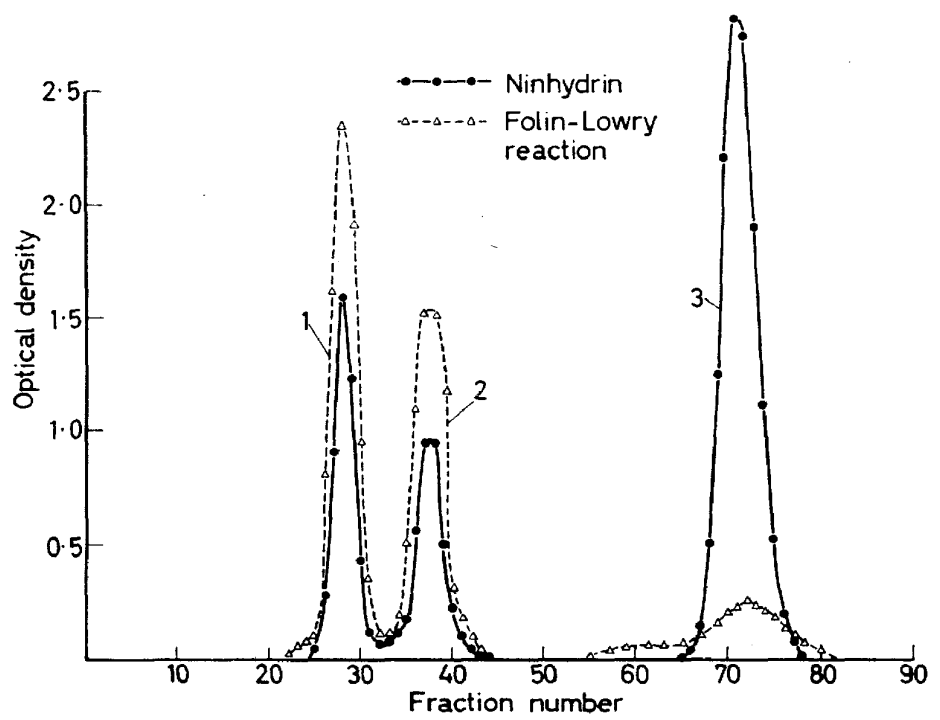

Figure 6. Ion-exclusion diagram of Fraction $E$ (Figure $3 b$ ). The first peak contains almost pure vasopressin; peak 2 , oxytocin

oxytocin, vasopressin and their analogues. For example, they should be particularly suited for the removal of linear oxidation products.

\section{PURIFICATION OF THYROTROPIC HORMONE (TSH)}

The thyroid stimulating factor of the anterior lobe of the pituitary has not yet been isolated, in spite of considerable efforts in many laboratories over a period of several years. Last year Condliffe and Bates of the National Institute of Arthritis and Metabolic Diseases at Bethesda approached us for collaboration. They had been able to obtain rather active material starting with bovine anterior lobe pituitary powder of a potency of 0.017 units per milligram ${ }^{9}$. Precipitation by $\mathrm{pH}$ adjustment and acetone in the cold yielded a product about 40 times as active as the starting material. Chromatography on carboxymethyl cellulose, CMC, followed by gel filtration on Sephadex $\mathrm{G} 50$ raised the potency to $c a .6$ units per milligram. The gel filtration diagram is given in Figure 7. Ammonium carbonate was used in the gel filtration experiment since this salt could be easily removed upon lyophilization leaving a salt-free protein preparation. The gel filtration on $G 50$ thus results in removal of a cytochrome $c$ component and the non-volatile salt introduced by the CMC-chromatography. The shape of TSH peak in the diagram probably reflects the presence of several complexes between TSH and other proteins in the medium used.

The partly purified TSH was chromatographed on DEAE-cellulose. A powder assaying 35 units per milligram was obtained. This preparation, when subjected to gel filtration on Sephadex G 100, gave the distribution curves shown in Figure 8 . The activity was located in the rear part of the protein peak. A considerable purification was achieved in this step. The material in the tubes 80 to 90 contained only 5 per cent of the original weight 


\section{J. PORATH}

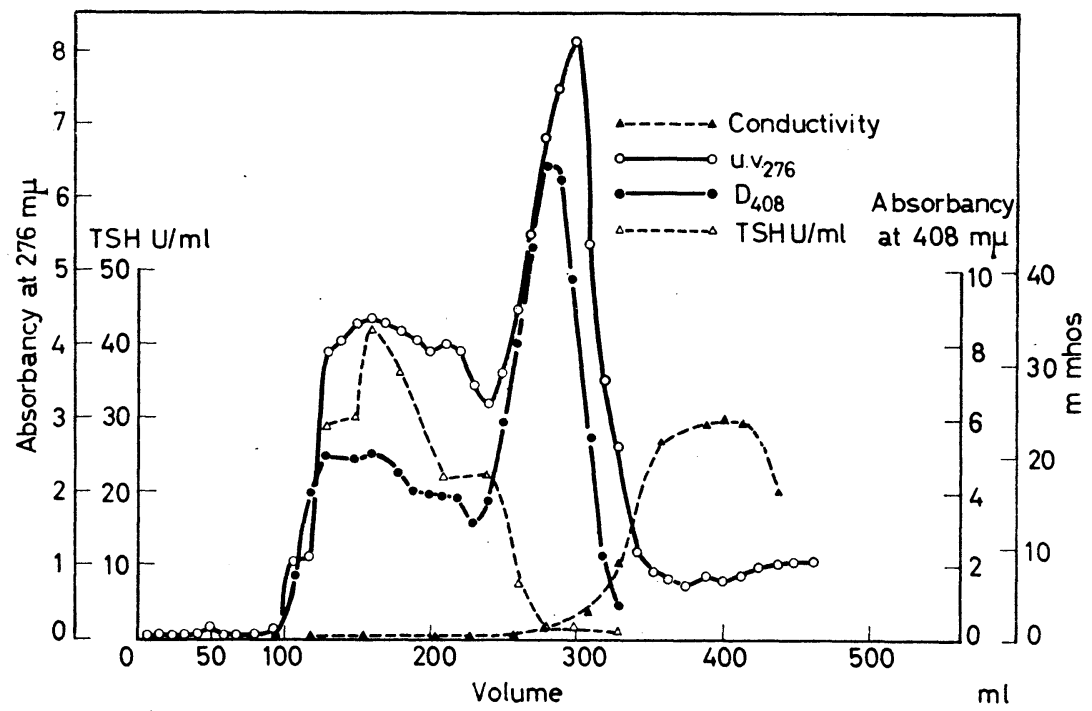

Figure 7. Fractionation of a crude TSH preparation on Sephadex G 50 (Condliffe and Bates)

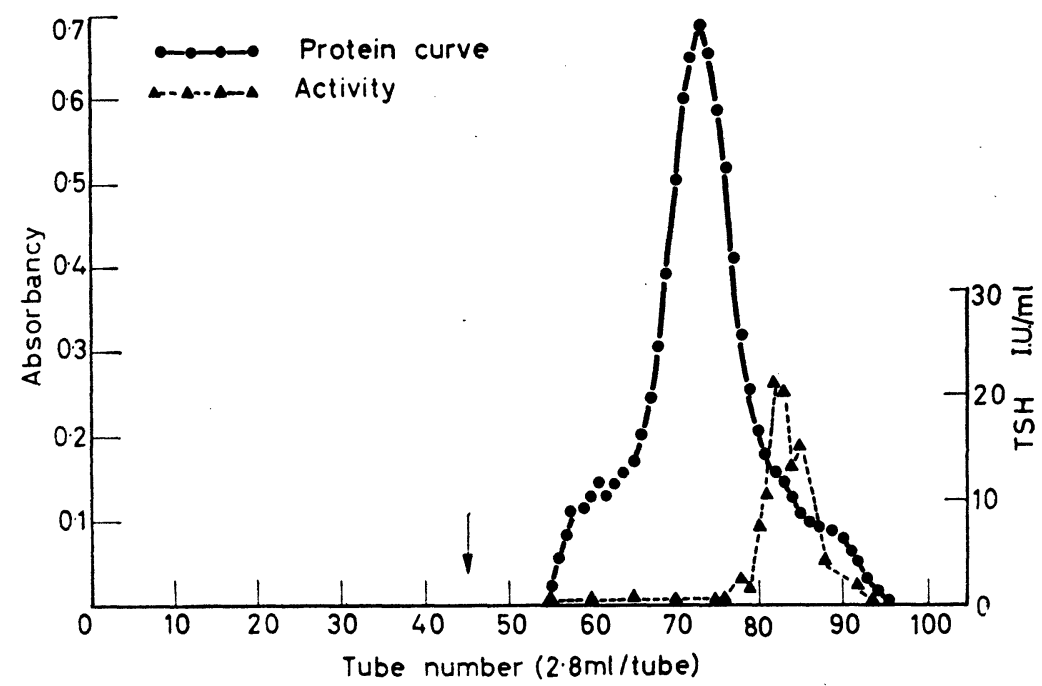

Figure 8 . Gel filtration diagram of a highly purified TSH preparation. Gel: Sephadex G 100 (Condliffe and Porath) ${ }^{12}$

of the protein and the activity was estimated to be over 200 units per milligram ${ }^{12}$.

The highly purified TSH preparations are very unstable. For this reason electrophoresis extended over long periods of time has not yet been successful in attempts aiming at final isolation.

Recently, Bennich and I have introduced a new method which we have 
called "recycling chromatography". The principle is similar to the recycling in counter-current liquid-liquid extraction and consists in circulating the solution many times through the column. The progressive fractionation of the solvent mixture is recorded by a suitable analyser (Figure 9). We hope to separate the bulk of protein from the active substance by this method.

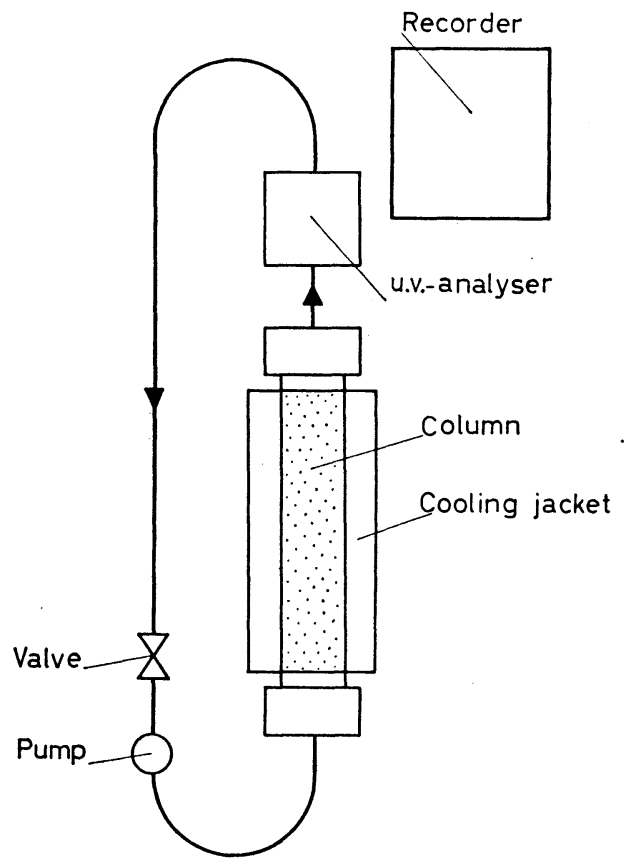

Figure 9. Schematic diagram showing the principle of recycling chromatography

Ceruloplasmin and carbonic anhydrase were filtered through the same column as was used in the experiment referred to in Figure 8. By means of these substances the column was calibrated with respect to molecular weight. The carbonic anhydrase and the thyrotropic activity were retarded to about the same extent. For this reason we believe that the molecular weight of the bovine thyrotropin is about 30,000 . This value is consistent with sedimentation analysis in a density gradient.

Gel filtration of mouse serum on Sephadex G 100 resulted in two main protein peaks as expected from the findings by Flodin and Killander ${ }^{13}$. The TSH activity was located in a region slightly behind the albumin. This suggests that TSH is present in free form in this serum and that mouse TSH is about the same molecular size as the bovine hormone.

\section{OTHER APPLICATIONS IN THE HORMONE FIELD}

Gel filtration may be used to fractionate serum as just mentioned. In fact it appears to be the most gentle method available at present for the purification of serum proteins. Together with Luft and Cjerassi we have 


\section{J. PORATH}

started an investigation of insulin in human serum. From our preliminary experiments we believe that insulin might be present in bound form and that the complex has a molecular weight of around 100,000 .

Rasmussen and Craig ${ }^{14}$ have recently been able to isolate pure peptides from bovine parathyroids which produce an elevation in the calcium concentration in the blood. They found Sephadex filtration to be a useful tool for this isolation.

There are many other applications to hormones but they are outside the scope of this paper. Those who are interested may find some of them treated in recent reviews ${ }^{15,16}$.

\section{References}

1 A. M. Biget. Ann. chim. (Paris) 5, 66 (1950).

2 J. Porath and P. Flodin. Nature 183, 1657 (1959).

3 B. H. Zimm and W. H. Stockmeyer. J. Chem. Phys. 17, 1301 (1949).

4 K. A. Granath and P. Flodin. Makromol. Chem. XLVIII, 160 (1961).

${ }^{5}$ P. Flodin and K. Aspberg. I.U.B./I.U.B.S. Symposium on Biological Structure and Function, Vol. 1, p. 365, Academic Press, London and New York (1960).

B J. Porath. Biochim. et Biophys. Acta 39, 193 (1960).

7 J. Chauvet, M.-T. Lenci, and R. Acher. Biochem. et Biophys. Acta 38, 266 (1960).

8 E.-B. Lindner, A. Elmqvist, and J. Porath. Nature 184, 1565 (1961).

${ }^{9} \mathrm{~J}$. Porath and E.-B. Lindner. Nature 191, 69 (1961).

${ }_{10}$ R. L. M. Synge and M. A. Youngson. Biochem. J. 78, 31 (1961).

11 J. Porath and A. V. Schally. Endocrinology 70, 738 (1962).

12 P. Condliffe and J. Porath. Federation Proc. A 199 b (1962).

13 P. Flodin and J. Killander. Biochim. et Biophys. Acta. In press.

14 H. Rasmussen and L. C. Craig. Biochim. et Biophys. Acta 56, 332 (1962).

13 P. Flodin and J. Porath. "Molecular sieve processes", in Chromatography, (Ed. E. Heftman) p. 328, Reinhold Publishing Corporation, New York (1961).

${ }_{16} \mathrm{~J}$. Porath. Advances in Protein Chem. In press. 